\title{
Production of a Functional Frozen Yogurt Fortified with Bifidobacterium spp.
}

\author{
Amro Abdelazez, ${ }^{1,2}$ Zafarullah Muhammad, ${ }^{1}$ Qiu-Xue Zhang, ${ }^{1}$ Zong-Tao Zhu, ${ }^{1}$ \\ Heba Abdelmotaal, ${ }^{3,4}$ Rokayya Sami, ${ }^{5,6}$ and Xiang-Chen Meng ${ }^{1}$ \\ ${ }^{1}$ Key Laboratory of Dairy Science of Ministry of Education, Northeast Agricultural University, Harbin 150030, China \\ ${ }^{2}$ Department of Dairy Microbiology, Animal Production Research Institute, Agriculture Research Center, \\ Dokki, Giza 12618, Egypt \\ ${ }^{3}$ Department of Microbiology, Soil, Water and Environment Research Institute, Agriculture Research Center, Giza 12619, Egypt \\ ${ }^{4}$ Department of Microbiology and Biotechnology, College of Life Sciences, Northeast Agricultural University, \\ Harbin 150030, China \\ ${ }^{5}$ Department of Nutrition and Food Science, Taif University, Taif, Al-Huwayah 888, Saudi Arabia \\ ${ }^{6}$ Department of Food Science, Northeast Agricultural University, Harbin, Heilongjiang 150030, China
}

Correspondence should be addressed to Xiang-Chen Meng; xchmeng@hotmail.com

Received 11 November 2016; Accepted 5 January 2017; Published 11 June 2017

Academic Editor: Pratik Banerjee

Copyright (C) 2017 Amro Abdelazez et al. This is an open access article distributed under the Creative Commons Attribution License, which permits unrestricted use, distribution, and reproduction in any medium, provided the original work is properly cited.

Frozen dairy products have characteristics of both yogurt and ice cream and could be the persuasive carriers of probiotics. Functions of the frozen yogurt containing viable bifidobacterial cells are recognized and favored by the people of all ages. We developed a kind of yogurt supplemented by Bifidobacterium species. Firstly, five strains of Bifidobacterium spp. (Bifidobacterium bifidum ATCC 11547, Bifidobacterium longum ATCC 11549, Bifidobacterium infantis ATCC 11551, Bifidobacterium adolescentis ATCC 11550, and Bifidobacterium breve ATCC 11548) were evaluated based on the feasibility criteria of probiotics, comprising acid production, bile tolerance, and adhesion to epithelial cells. Formerly, we combined the optimum strains with yogurt culture (Lactobacillus delbrueckii subsp. bulgaricus EMCC 11102 and Streptococcus salivarius subsp. thermophilus EMCC 11044) for producing frozen yogurt. Finally, physiochemical properties and sensory evaluation of the frozen yogurt were investigated during storage of 60 days at $-18^{\circ} \mathrm{C}$. Results directed that Bifidobacterium adolescentis ATCC 11550 and Bifidobacterium infantis ATCC 11551 could be utilized with yogurt culture for producing frozen yogurt. Moreover, the frozen yogurt fermented by two bifidobacterial strains and yogurt culture gained the high evaluation in the physiochemical properties and sensory evaluation. In summary, our results revealed that there was no significant difference between frozen yogurt fermented by Bifidobacterium spp. and yogurt culture and that fermented by yogurt culture only.

\section{Introduction}

Diet plays an important role in preventing diseases and ensuring health. Hence, the consumption of functional foods (i.e., beneficial compounds or foods containing microorganisms) which provide health benefits with a reduction of coronary heart disease, obesity risk, and diabetes has increased during the last decade [1]. The concept of using probiotics to improve and maintain human health is not new at all. Probiotic microorganisms are usually used as culture concentrates in dried or deep-freeze forms to be added to food for industrial or home uses [2]. In addition to the probiotic foods, there are various health products and pharmaceutical preparations containing probiotics on the market [3].

Bifidobacterium is an important group of probiotic cultures and commonly used in fermented dairy products that contributes a major part in the human intestinal microbiota in healthy humans. They are considered to provide 
many beneficial effects including improvement of lactose digestibility, anticarcinogenic activity, reduction of serum cholesterol level, synthesis of B vitamins, and facilitation in calcium absorption [4]. Moreover, numerous studies with different strains of Lactobacillus and Bifidobacterium have been performed in vitro and in vivo, in humans and animal models to investigate their immunomodulatory properties and probiotic potential to treat various infectious, allergic, and inflammatory conditions [5, 6]. Even though Bifidobacterium strains have already been used in dairy products, they have some inferior behavioral characteristics compared with the traditional lactic acid bacteria (LAB) used in fermented dairy products, hindering their possible applications [7]. Vitally, they represent weaker growth and acid production in cow milk and require long fermentation times, anaerobic conditions, and low redox potential for their growth [8].

There are clear relationship between the food we eat and our health. Therefore, some reports have investigated ice cream and yogurt as probiotic carrier. Hence, frozen yogurt is a novel way of combining the characteristics of ice cream with the therapeutic properties of yogurt that are considered as a healthy alternative to ice cream for the people suffering from cardiovascular diseases and lactose intolerance [1, 912]. The aim of study was to examine different factors affecting survival and activity of five species of bifidobacteria, study the viability of two chosen Bifidobacterium species in manufactured frozen yogurt under different conditions, and investigate the effect of storage temperatures on their viability.

\section{Materials}

2.1. Additives. Skim milk powder, vanilla, and sugar were purchased from local market. Stabilizer, emulsifier, and Cremondan SE 38 veg were provided by Danisco Ingredients, Denmark.

\subsection{Bacterial Strains. Freeze dried Lactobacillus delbrueckii} subsp. bulgaricus EMCC 11102 and Streptococcus salivarius subsp. thermophilus EMCC 11044 and Bifidobacterium species including Bifidobacterium bifidum ATCC 11547, Bifidobacterium longum ATCC 11549, Bifidobacterium infantis ATCC 11551, Bifidobacterium adolescentis ATCC 11550, and Bifidobacterium breve ATCC 11548 were provided by Cairo Microbiological Resources Center, Egypt.

\section{Methods}

3.1. Determination of Maximum Growth Rate and Maximum Acidification of Bifidobacterium spp. Strains in MRSL. Bifidobacterium spp. were inoculated (1\% v/v) and grown in MRSL (Man Rogosa Sharpe) broth (Oxoid, Basingstoke, UK) supplemented with 5\% (w/v) lactose (Win Lab, Gemini House, Middlesex, Hab 7ET, UK) and 0.05\% (w/v) Lcysteine-HCL (Merck, Germany) at $37^{\circ} \mathrm{C}$ under anaerobic conditions (BBL Gas Pak, Becton Dickinson, Cockeysville MA, USA). The bacterial growth was monitored by measuring the absorbance with a spectrophotometer (DU 800, Beckman Coulter, USA) at $660 \mathrm{~nm}$. Moreover, $\mathrm{pH}$ was determined by using $\mathrm{pH}$ meter (MP 220, Metler Toledo, Greifensee, Switzerland). The maximum acidification rate was reported according to [13].

3.2. Bile Salts Tolerance of Bifidobacterium spp. According to [14] Bifidobacterium spp. strains were inoculated in MRSL broth added to $0.3 \%(\mathrm{w} / \mathrm{v})$ oxgall powder (Merck, Germany) and incubated at $37^{\circ} \mathrm{C}$ under anaerobic conditions for $24 \mathrm{hr}$. Bacterial growth was monitored by measuring absorbance with a spectrophotometer at $660 \mathrm{~nm}$ after $24 \mathrm{hr}$. The obtained absorbance values were plotted against the incubation time. Strain inoculated in MRSL broth without oxgall powder was taken as the control. Correlation between all the results of Bifidobacterium spp. resistance to bile salts was determined by the principal component analysis (PCA) using XLSTAT software.

3.3. Calculation of Survival Rate in Bile Salts. The survival rate was calculated by using the following formula reported by [15]:

$$
\% \text { Bile survival }=\frac{\log N_{1}}{\log N_{0}} \times 100
$$

$\log N_{1}$ is absorbance of culture in MRSL broth containing $0.3 \%$ bile salts.

$\log N_{0}$ is absorbance of culture in MRSL broth without bile salts.

3.4. Adhesion of Bifidobacterium spp. to Intestinal Epithelial Cells. According to [13] for the adherence assay, five Bifidobacterium spp. strains were tested for the adherence to epithelial cells. Bifidobacterium spp. strains were inoculated in MRSL broth and incubated overnight at $37^{\circ} \mathrm{C}$ under anaerobic conditions. The cultures were adjusted overnight to 1.5 $\times 10^{8} \mathrm{CFU} / \mathrm{ml}$ and then $10 \mathrm{ml}$ of Bifidobacterium spp. cultures was removed and centrifuged at $4000 \times \mathrm{g}$ RPM for $12 \mathrm{~min}$. The supernatant was discarded, and $10 \mathrm{ml}$ PBS (pH 7.2) was added and mixed using vortex. The crop scraping of epithelial cells was prepared by scrapping off the epithelium from rabbit duodenum with the edge of a microscope slide, washed by phosphate buffered saline, and suspended in buffer ( $\mathrm{pH} 7.2)$. Moreover, cell cultures were washed five times with sterile phosphate buffered saline (PBS) ( $\mathrm{pH} 7.2$ ). Thereafter, $0.4 \mathrm{ml}$ of epithelial cell suspension was added to $0.1 \mathrm{ml}$ of bacterial cell suspension. The mixture was centrifuged at $4000 \times \mathrm{g} \mathrm{RPM}$ for $5 \mathrm{~min}$ and then incubated at $37^{\circ} \mathrm{C}$ for $30 \mathrm{~min}$. Finally, binding between the bifidobacterial cells and epithelial cells was examined by gram stained phase contrast microscopy (magnification fold, 200x). The adhered bifidobacterial cells were determined by counting adhering bifidobacterial cells in 15 randomly selected microscopic fields.

\subsection{Manufacturing Procedure of Frozen Yogurt}

3.5.1. Preparation of Yogurt. Experimental plain yogurt was prepared by heating pasteurized whole milk at $72^{\circ} \mathrm{C}$ for 10 
minutes and subsequently cooled to $43^{\circ} \mathrm{C}$ Then, it was divided into five separate containers:

Formula 1 (C) inoculated with $1 \%$ w/w starter yogurt culture with no Bifidobacterium spp.

Formula $2(C+A)$ inoculated with $1 \%$ w/w starter yogurt culture $+1 \% \mathrm{w} / \mathrm{w}$ of $B$. adolescentis.

Formula $3(C+B)$ inoculated with $1 \%$ w/w starter yogurt culture $+1 \% \mathrm{w} / \mathrm{w}$ of $B$. infantis.

Formula $4(A+B)$ inoculated with $1 \% \mathrm{w} / \mathrm{w}$ B. adolescentis $+1 \% \mathrm{w} / \mathrm{w}$ of $B$. infantis.

Formula $5(C+A+B)$ inoculated with $1 \%$ w/w starter yogurt culture $+1 \% \mathrm{w} / \mathrm{w} B$. adolescentis $+1 \% \mathrm{w} / \mathrm{w}$ of $B$. infantis.

The inoculated mixtures were incubated at $37^{\circ} \mathrm{C}$ until the pH 5.9 was obtained.

3.6. Preparation of Frozen Yogurt. Five frozen yogurt blends, each of three replicates, were prepared. All mixtures were standardized to contain $8 \%$ fat, $12 \%$ milk solids not fat, $16 \%$ sugar, $0.8 \%$ stabilizer/emulsifier, and $0.3 \%$ vanilla. In each treatment, mixed ingredients were homogenized together by using the method described by [16] with some modifications and then heated at $80^{\circ} \mathrm{C}$ for $30 \mathrm{~min}$. All mixes were cooled at $5^{\circ} \mathrm{C}$ and then aged overnight at the same temperature. On the other hand, prepared yogurt was added $(10 \% \mathrm{v} / \mathrm{v})$ to five ice cream mixes prior to freezing. The freezing was performed in a horizontal batch freezer (Taylor Co., USA) and hardened at $-18^{\circ} \mathrm{C}$ for $24 \mathrm{~h}$ before analyses.

3.7. Physicochemical Analyses. Frozen yogurt samples were stored at $-18 \pm 2^{\circ} \mathrm{C}$ for 60 days, and the physicochemical analyses were performed at $0,15,30$, and $60 \mathrm{~d}$. Titratable acid (TA) and total solid (TS) were analyzed for all frozen yogurt samples according to [17], and $\mathrm{pH}$ was determined by $\mathrm{pH}$ meter (MP 220, Metler Toledo, Greifensee, Switzerland).

3.8. Overrun and Meltdown Tests. The overrun was calculated according to [17].

$$
\text { Overrun }=\left[\frac{(W 1-W 2)}{W 2}\right] \times 100,
$$

where $W 1$ is weight of the mix and $W 2$ is weight of the same volume of frozen yogurt. The meltdown test was conducted in a chamber with controlled temperature $\left(25^{\circ} \mathrm{C}\right)$. According to the method described by [18]. Results were expressed as a time for collection of each $10 \mathrm{ml}$ of liquid.

3.9. Hardness. Texture analysis was performed using Texture Analyzer (TA.XT Plus Texture Analyzer, UK). The samples were stored in $50 \mathrm{~mm}$ plastic containers at $-18^{\circ} \mathrm{C}$ until analysis. Measurement was carried out by using a cylindrical probe. Penetration depth at the geometrical center of the sample was $10 \mathrm{~mm}$ and penetration speed was set at $2 \mathrm{~mm} / \mathrm{s}$. The hardness was determined as the peak compression force (g) during penetration [19].

3.10. Enumeration of Viable Bifidobacterium spp. in Frozen Yogurt. The viable bifidobacterial cell count in frozen yogurt samples containing Bifidobacterium spp. was determined and expressed as colony forming units $(\mathrm{CFU} / \mathrm{mL})$ during storage of $0,15,30$, and $60 \mathrm{~d}$ at $-18 \pm 2^{\circ} \mathrm{C}$. Bifidobacterial cell counts were enumerated on MRSL agar using pour plate technique. The plates were incubated anaerobically at $37^{\circ} \mathrm{C}$ for $72 \mathrm{hr}$. Survival rates percentage of bifidobacteria was calculated according to [20].

3.11. Sensory Appraisal. Organoleptic properties of frozen yogurt were evaluated after 60 days of storage according to [21], for flavor (45 points), body and texture (35 points), appearance (10 points), melting quality (10 points), and total scores (100 points) by 20 panelists of the experienced staff members of the Dairy Science Department, Faculty of Agriculture, Minia University, Egypt.

3.12. Statistical Analysis. All experiments and analyses were performed in triplicate. The results were given as means \pm the standard error of mean (SEM) and analyzed by using Graph Pad Prism 5 software. Comparisons between groups were performed by using one-way analysis of variance (ANOVA) after $t$-test. In addition, $p<0.05$ was considered significant. The PCA using XLSTAT software determined the correlation between all the experiments.

\section{Results and Discussions}

4.1. Growth Rate and pH of Bifidobacterium spp. in MRSL at $37^{\circ} \mathrm{C}$. All the bifidobacterial species showed a similar growth profile when Bifidobacterium spp. were incubated in MRSL at $37^{\circ} \mathrm{C}$. The first log phase was observed during the first 12 to $24 \mathrm{hr}$ of growth and second log phase was started at $48 \mathrm{hr}$ and continued until $56 \mathrm{hr}$ and after that decline phase was started (Figure 1(a)).

The kinetics growth of five Bifidobacterium spp. and $\mathrm{pH}$ investigated that $B$. adolescentis, B. breve, and B. longum grown well in lactose MRS and the rates of growth were $1.363,1.362$, and 1.223 at $65 \mathrm{hr}$, respectively, at log phase, while results in Figure 1(b) have shown the decrease of $\mathrm{pH}$ gradually from 5.48 at zero time to $3.41,3.56$, and 3.63, respectively, after $65 \mathrm{hr}$. However, growth of the $B$. adolescentis, B. breve, and B. longum was $0.937,0.935$, and 0.907 at $96 \mathrm{hr}$, respectively, whereas $\mathrm{pH}$ was $2.98,3.36$, and 3.26 at $96 \mathrm{hr}$, respectively. On the contrary, growth of the B. infantis and B. bifidum was 1.183 and 1.164 at $65 \mathrm{hr}$ of incubation and $\mathrm{pH}$ was 3.52 and 3.53, respectively. Meanwhile, the growth was 0.839 and 0.935 and $\mathrm{pH} 3.23$ and 3.24 , respectively, at $96 \mathrm{hr}$. These results were in complete consensuses with [13] that have attributed this pattern of growth to the presence of two different $\beta$-galactosidases. However, B. adolescentis showed the highest growth rate, followed by $B$. breve and B. bifidum. Meanwhile, $B$. infantis and B. longum were the lowest at $65 \mathrm{hr}$ of incubation. Moreover, the differences in growth rate among species of Bifidobacterium spp. correlated to different levels of tolerance to aerobic conditions.

4.2. Resistance of Bifidobacterium spp. to Bile Salts in MRSL Incubated at $37^{\circ} \mathrm{C}$. Bile tolerance is one of the most crucial 


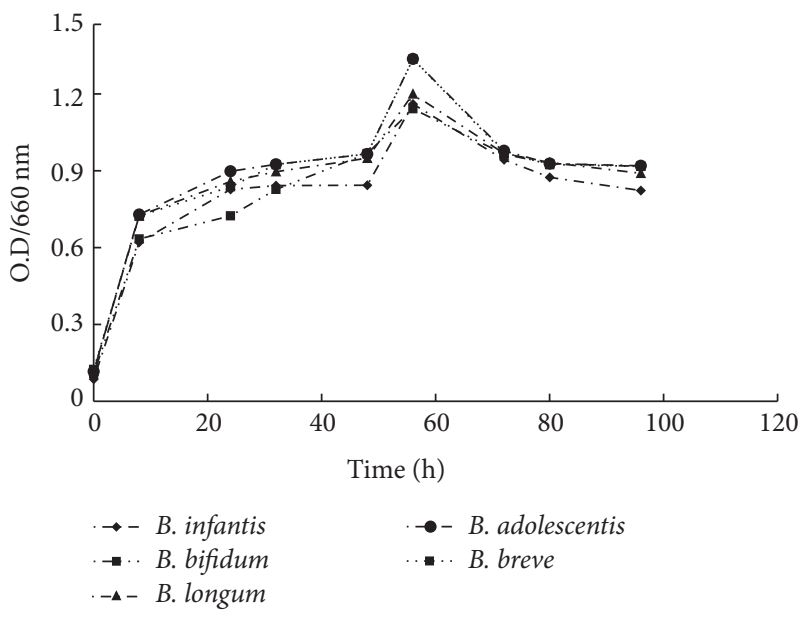

(a)

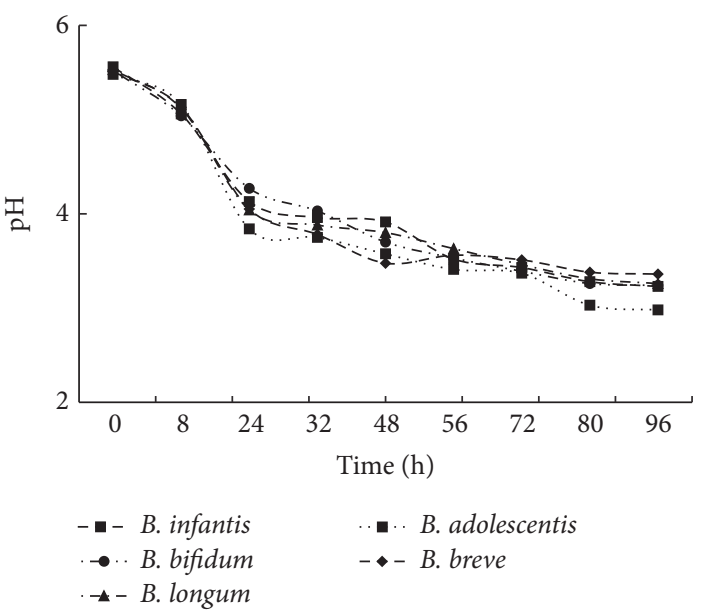

(b)

FIGURE 1: (a) Growth rate of different species of Bifidobacterium spp. (b) pH of different species of Bifidobacterium spp.

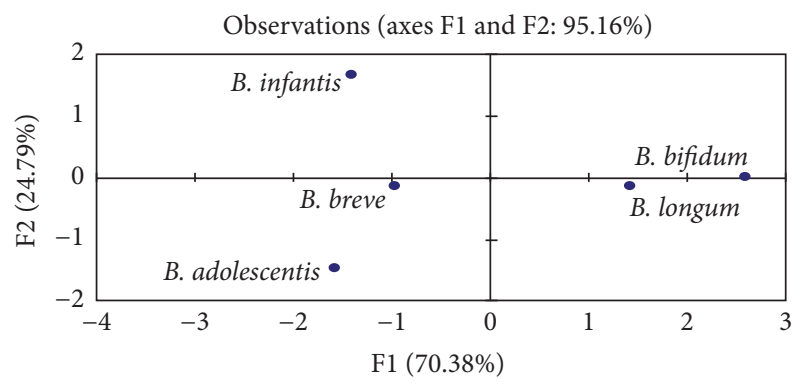

FIGURE 2: Plots of the $x$-loadings of Bifidobacterium spp.

properties as it determines the ability of bacteria to survive in the small intestine and play their functional role as probiotics. A concentration of $0.3 \%$ of bile salts closely appropriates the bile level, which are found in the gastrointestinal tract [22]. Common observations among this comparison of different cultures for bile salts tolerance were shown in this study. The highest and lowest resistance of five Bifidobacterium spp. were observed in Figure 4. It was shown that $B$. infantis and $B$. bifidum were more resistant to bile salts than the other three species that they reached O. $\mathrm{D}_{660}$ of 0.82 and 0.61 at $24 \mathrm{hr}$, respectively. On the contrary, $B$ adolescentis had a dramatically decreased O.D $\mathrm{D}_{600}$ of 0.31 at $24 \mathrm{hr}$ according to these results. Finally, we summarized that the growths of Bifidobacterium spp. were harmed by bile salts. Moreover, these results were in convergence with [23] who reported the tolerance of Bifidobacterium to bile or acid. Therefore, $B$. infantis had the highest survival rates followed by $B$. bifidum, $B$. breve, and B. longum, when exposed to bile salts at concentrations ranging from zero to $3 \mathrm{~g} / \mathrm{L}$.

The result of the PCA was used to study the resistance of Bifidobacterium spp. to bile salts. Figures 2 and 3 presented the plots of the scores and the correlation loadings, respectively. The score plots of PCA illustrated the large variability of the five Bifidobacterium spp. based on their resistance to bile salts. The loadings are the coefficients of the original variables

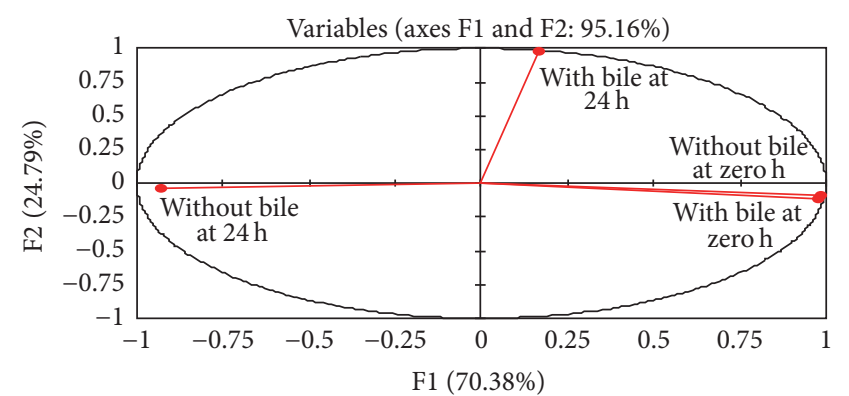

Figure 3: Plots of the scores of Bifidobacterium spp.

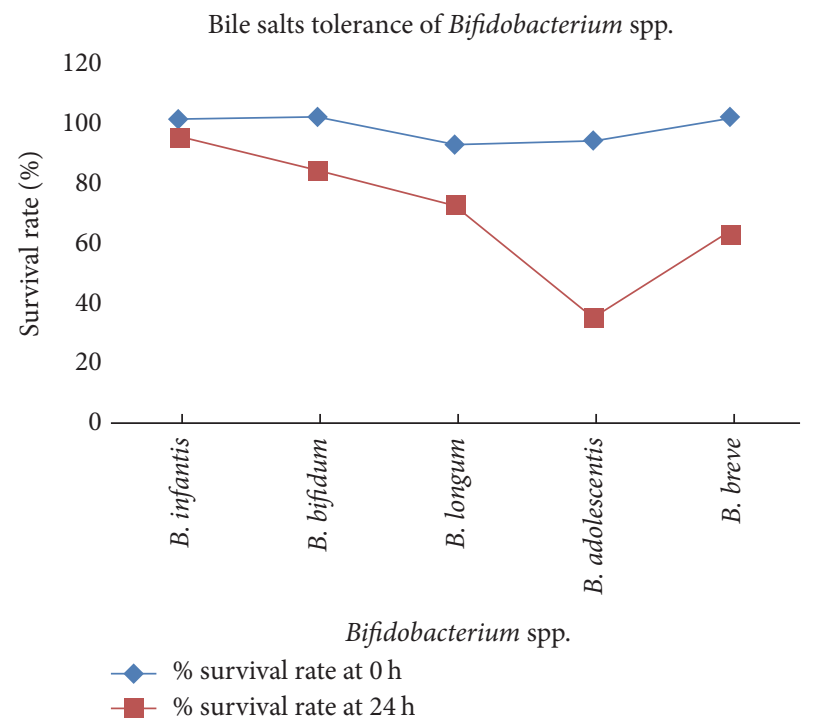

FIgURE 4: Resistance percentage of Bifidobacterium spp. to bile salts.

that define each principal component. Inertia percentage and correlated variables for axes 1 and 2 were displayed in Table 1. Axis 1 explained $70.38 \%$ of the total inertia. Axis 2 explained $24.79 \%$ of the inertia. Plots of the scores in Figure 2 indicated 


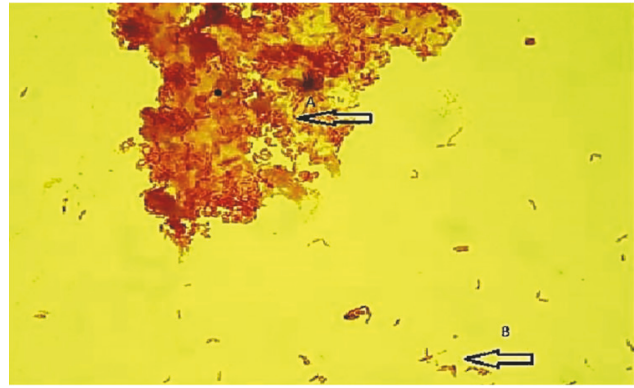

B. adolescentis

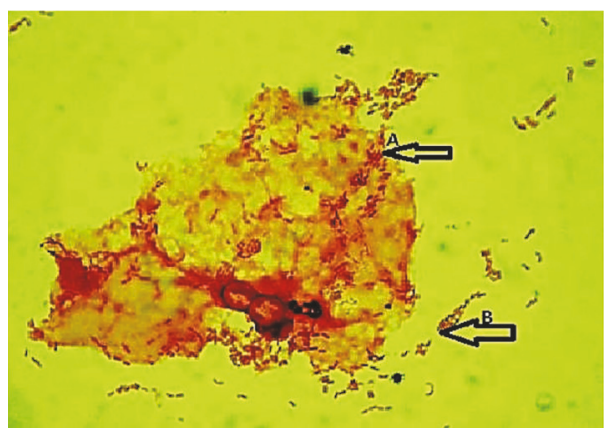

B. bifidum

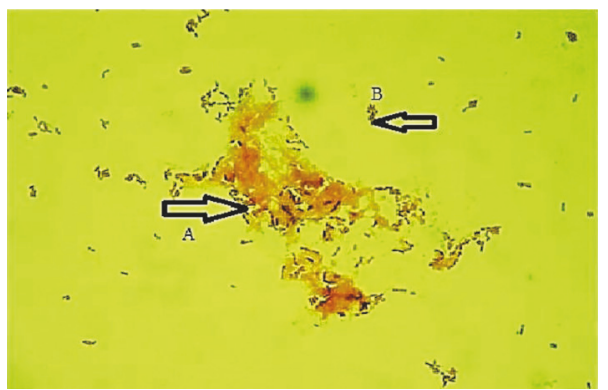

B. breve

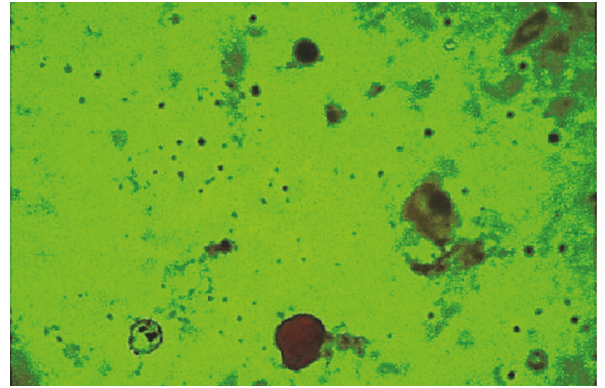

Control

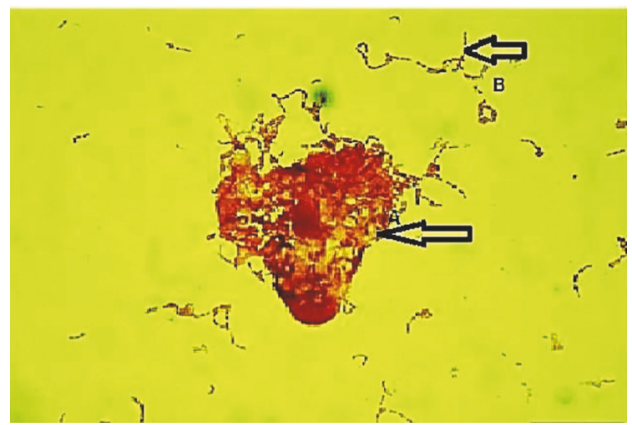

B. longum

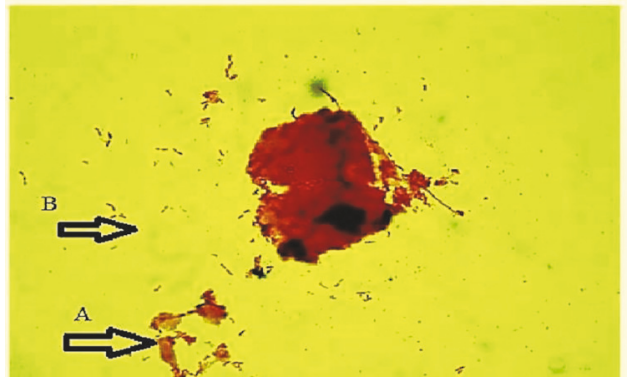

B. infantis

$\mathrm{A}=$ adhesion Bifidobacterium, $\mathrm{B}=$ nonadhesion Bifidobacterium

Figure 5: Adhesion of Bifidobacterium spp. to intestinal epithelial cells.

TABLE 1: Discriminate variables factors of principal components analysis to study the resistance of Bifidobacterium spp. to bile salts.

\begin{tabular}{lcc}
\hline & F1 & F2 \\
\hline Proper value & 2.82 & 0.99 \\
Variability (\%) & 70.38 & 24.79 \\
Cumulative (\%) & 70.38 & 95.17 \\
\hline
\end{tabular}

that the data cloud was mainly bidimensional with respect to the explanatory variables. Figure 3 showed three clusters of Bifidobacterium spp. First cluster included the B. breve and $B$ adolescentis species. Second cluster included the B. bifidum and B. longum species. The third cluster (B. infantis species) was individualized.

4.3. Adhesion of Bifidobacterium spp. to Intestinal Epithelial Cells. Major considerations in the choice of Bifidobacterium spp. to be used as dietary adjuncts are not only the capability of survival and passing the harmful GI conditions, but also being established within the digestive tract. Caco-2 cells are human intestinal cell lines expressing morphologic and physiologic characteristics of normal human enterocytes [24]. That has been exploited to select and assess probiotics based on their adhesion properties.

Therefore, the adhesion of Bifidobacterium spp. to columnar epithelial cells of the small intestine of rabbit was tested as shown in Figure 5. It appeared that the ability of adhesion by $B$. adolescentis to Caco- 2 cells was stronger than that of other tested strains, but mainly with resistance to bile salts. In contrary, $B$. infantis was less capable of adhering to epithelial cells and acid production, but it was the best strain resistant to bile salts.

According to data shown in Figures 1(a), 1(b), 4, and 5 B. adolescentis have the highest percentage in survival rate at low $\mathrm{pH}$ and stronger adhesion to the epithelial cells. Meanwhile, B. infantis is best strain in resistance of bile salts. Therefore, we have chosen these strains to manufacture frozen yogurt. 


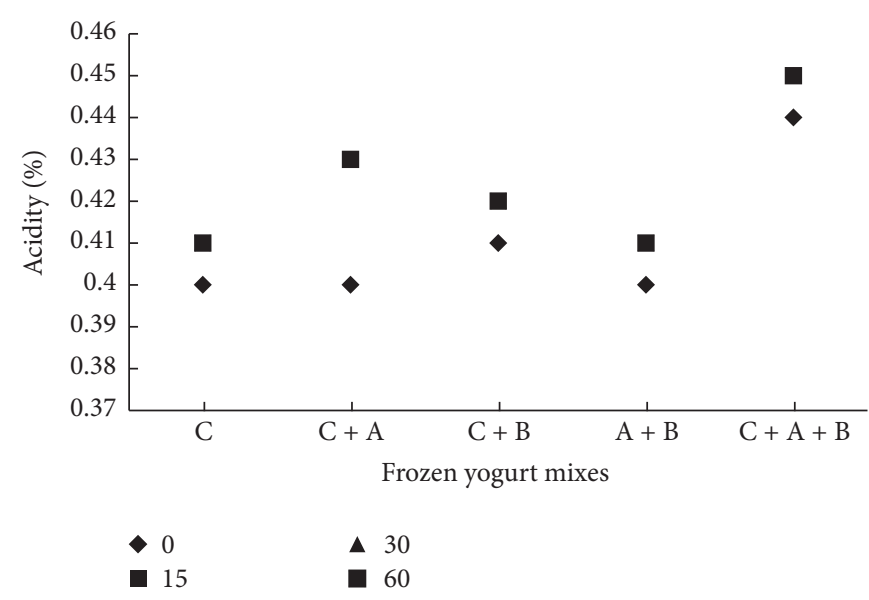

(a)

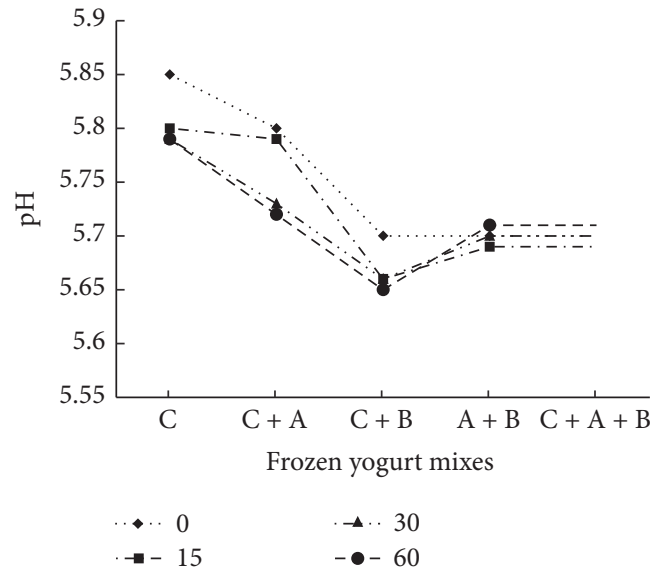

(b)

FIGURE 6: (a) Changes in titratable acidity of frozen yogurt during storage of $0,15,30$, and $60 \mathrm{~d}$ at $-18^{\circ} \mathrm{C}$. (b) Changes in $\mathrm{pH}$ of frozen yogurt during storage of $0,15,30$, and $60 \mathrm{~d}$ at $-18^{\circ} \mathrm{C}$.

4.4. Physicochemical Characteristics of Frozen Yogurts during 60 Days of Storage at $-18^{\circ} \mathrm{C}$ Acidity and $\mathrm{pH}$. These studies were conducted to see changes in acidity, $\mathrm{pH}$, and total solids of frozen yogurt made with yogurt culture and Bifidobacterium spp. during 60 days of storage at $-18^{\circ} \mathrm{C}$.

Results indicate that there are similar changes of titratable acidity and $\mathrm{pH}$ values development observed in different frozen yogurt treated. Only slight changes were found in mix $(\mathrm{C}+\mathrm{A}+\mathrm{B})$, where the acidity was increased to reach 0.45 at 60 days in the end of storage period. Furthermore, acidity development and $\mathrm{pH}$ were steady for five treatments of 60 days of storage. No significant differences $(p>0.05)$ in titratable acidity and $\mathrm{pH}$ values were noted among different frozen yogurt mixes during storage periods. These results indicated that the addition of Bifidobacterium had no obvious changes.

Data in Figures 6(a) and 6(b) were in conformity with the results obtained by [25], who found that titratable acidity of fresh frozen yogurt made with yogurt culture or Bifidobacterium spp. culture was 0.45 . These indicated that there were no biochemical activities by yogurt culture during storage of the product at $-20^{\circ} \mathrm{C}$. On the contrary, these results were in disagreement with findings by [26] who reported that the addition of the Bifidobacterium spp. led to lower $\mathrm{pH}$.

4.5. Total Solids. Total solids play an important role in the quality of frozen yogurt. Results of frozen yogurt samples made with yogurt culture and Bifidobacterium spp. during 60 days of storage at $-18^{\circ} \mathrm{C}$ indicated that total solids in all treatments made with yogurt culture and Bifidobacterium spp. were about 25.54 to 26.10 . These results demonstrated that there was no high significance at $p<0.05$, among frozen yogurt samples during the storage periods. Therefore, these have close conformities with results obtained by [27], who found that total solids of frozen yogurt made with yogurt culture and Bifidobacterium spp. culture with up to 5 weeks of storage at $-25^{\circ} \mathrm{C}$ did not significantly changed. Moreover, [28] reported that a slight increase in total solids was found in all samples during storage period up to 60 days. They attributed an increase to the partial losses in free water during storage.

\subsection{Changes in Rheological Properties of Frozen Yogurt Made with Yogurt Culture and Bifidobacterium spp. during 60 Days of Storage at $-18^{\circ} \mathrm{C}$}

4.6.1. Changes in Hardness ( $g$ ) of Frozen Yogurt during 60 Days of Storage at $-18^{\circ} \mathrm{C}$. As seen in Table 2 hardness of frozen yogurt made with yogurt culture only $(C)$ was $75-85$ while hardness of frozen yogurt made with Bifidobacterium spp. (C $+A),(C+B)$, and $(A+B)$ was 95,90 , and 88 , respectively, at 60 days of storage. On the contrary, hardness of mix (C $+\mathrm{A}+\mathrm{B}$ ) was the highest values; it was 85 at fresh samples and $88.33,96$, and 98 for 15,30 , and 60 days' storage period, respectively. These obtained results were in agreement with results obtained by [29] who reported that no significant ( $p$ $<0.05$ ) differences in hardness were found between frozen yogurts samples. Therefore, the addition of Bifidobacterium spp. did not affect the texture of the frozen yogurt.

4.6.2. Changes in Meltdown/Min of Frozen Yogurt during 60 Days of Storage at $-18^{\circ} \mathrm{C}$. Results in Table 2 showed that the meltdown of frozen yogurt made of yogurt culture (C) was in the range from 116.3 to $265.1 \mathrm{~min}$ of fresh to 60 days' storage at $-18^{\circ} \mathrm{C}$, while the time for collection was increased in mixed yogurt culture $+B$. adolescentis $(\mathrm{C}+\mathrm{A})$ from 113.1 to 124.2 of fresh to 60 days' storage at $-18^{\circ} \mathrm{C}$. Moreover, frozen yogurt made with yogurt culture $+B$. infantis $(C+B)$ was slightly decreased from 138.1 to 124.3. In addition, Bifidobacterium spp. culture $(A+B)$ mix was in the range from 286.3 to 275.9 min of fresh to 60 days' storage at $-18^{\circ} \mathrm{C}$. Therefore, frozen yogurt made with three combinations of cultures $(\mathrm{C}+\mathrm{A}+$ B) had dramatically increased from 107.2 to 130 from fresh to 60 days of storage at $-18^{\circ} \mathrm{C}$. Finally, we summarized that only slight changes were found in mix $(C+A+B)$ which 
TABLE 2: Changes in some rheological properties of frozen yogurt made with yogurt culture and Bifidobacterium spp. culture during 60 days of storage $-18^{\circ} \mathrm{C}$.

\begin{tabular}{|c|c|c|c|c|}
\hline Treatment & Storage time/days & Hardness/g & Meltdown/min & \% Overrun \\
\hline \multirow{4}{*}{$\mathrm{C}$} & 0 & $75 \pm 1^{j}$ & $116.3 \pm 1^{\circ}$ & \multirow{4}{*}{24.5} \\
\hline & 15 & $78.58 \pm 0.1^{\mathrm{hi}}$ & $194.8 \pm 1^{\mathrm{e}}$ & \\
\hline & 30 & $80 \pm 1^{\mathrm{h}}$ & $188 \pm 1^{\mathrm{f}}$ & \\
\hline & 60 & $85 \pm 1^{\mathrm{f}}$ & $265.1 \pm 1^{\mathrm{d}}$ & \\
\hline \multirow{4}{*}{$\mathrm{C}+\mathrm{A}$} & 0 & $82.07 \pm 1^{g}$ & $113.1 \pm 1^{p}$ & \multirow{4}{*}{42.86} \\
\hline & 15 & $87 \pm 1^{\mathrm{e}}$ & $135 \pm 1^{\mathrm{h}}$ & \\
\hline & 30 & $92 \pm 1^{\mathrm{c}}$ & $128 \pm 1^{j}$ & \\
\hline & 60 & $95 \pm 1^{b}$ & $124.2 \pm 1^{1}$ & \\
\hline \multirow{4}{*}{$C+B$} & 0 & $78 \pm 1^{\mathrm{i}}$ & $138.1 \pm 1^{g}$ & \multirow{4}{*}{43} \\
\hline & 15 & $80 \pm 1^{\mathrm{h}}$ & $120.5 \pm 1^{\mathrm{m}}$ & \\
\hline & 30 & $87 \pm 1^{\mathrm{e}}$ & $118 \pm 1^{\mathrm{n}}$ & \\
\hline & 60 & $90 \pm 1^{\mathrm{d}}$ & $124.3 \pm 1^{1}$ & \\
\hline \multirow{4}{*}{$A+B$} & 0 & $76 \pm 1^{j}$ & $286.3 \pm 1^{b}$ & \multirow{4}{*}{43.7} \\
\hline & 15 & $79 \pm 1^{\mathrm{hi}}$ & $323 \pm 1^{\mathrm{a}}$ & \\
\hline & 30 & $83 \pm 1^{g}$ & $276 \pm 1^{c}$ & \\
\hline & 60 & $88 \pm 1^{\mathrm{e}}$ & $275.9 \pm 1^{\mathrm{c}}$ & \\
\hline \multirow{4}{*}{$C+A+B$} & 0 & $85.05 \pm 1^{\mathrm{f}}$ & $107.2 \pm 1^{\mathrm{q}}$ & \multirow{4}{*}{44.5} \\
\hline & 15 & $88.33 \pm 1.26^{\mathrm{e}}$ & $126 \pm 1^{\mathrm{k}}$ & \\
\hline & 30 & $96 \pm 1^{\mathrm{b}}$ & $120.1 \pm 1^{\mathrm{m}}$ & \\
\hline & 60 & $98 \pm 1^{\mathrm{a}}$ & $130 \pm 1^{\mathrm{i}}$ & \\
\hline
\end{tabular}

Values are the average of three individual samples each analyzed in duplicate \pm standard deviation. Different lowercase superscript letters, respectively, indicate significant difference $(p<0.05)$ analyzed by Duncan's multiple range test.

increased in meltdown/min of frozen yogurt. Moreover, it was clear that there was no significant $(p>0.05)$ difference in melting time and overrun values between different frozen yogurt mixes. The melting behavior of the product coincided with previous reports focusing on the melting behavior of ice cream with and without probiotics [30]. These findings were in close agreement with the findings of [31].

4.6.3. Changes in Overrun Percentage of Frozen Yogurt Made with Different Bifidobacterium spp. Overrun is one of the most important quality parameters of frozen desserts, since it affects the texture and consequently the price of the products. Results in Table 2 showed that the overrun levels of the five studied frozen yogurt formulations were low $(42.5 \%-44.50 \%)$ and these results were in contrast to [32], who reported that the addition of Bifidobacterium spp. led to no high changes in the overrun levels $(p<0.05)$. We hypothesized that it would lead to a poorer foaming capacity and decrease air incorporation in samples with blending components.

4.6.4. Changes in the Viability of Bifidobacterium spp. in Frozen Yogurt during 60 Days of Storage at $-18^{\circ} \mathrm{C}$. Results in Figure 7 showed the revealed count of Bifidobacterium spp. at $-18^{\circ} \mathrm{C}$ decreased with storage period. The count of Bifidobacterium spp. for frozen yogurt made with yogurt culture and $B$. adolescentis $(\mathrm{C}+\mathrm{A})$ was from $2.6 \times 10^{8}$ to $0.75 \times 10^{8} \mathrm{CFU}$ with decrease percent $71.20 \%$ from fresh to 60 days of storage period, while that of frozen yogurt made with yogurt culture and $B$. infantis $(C+B)$ was from $2.73 \times 10^{8}$

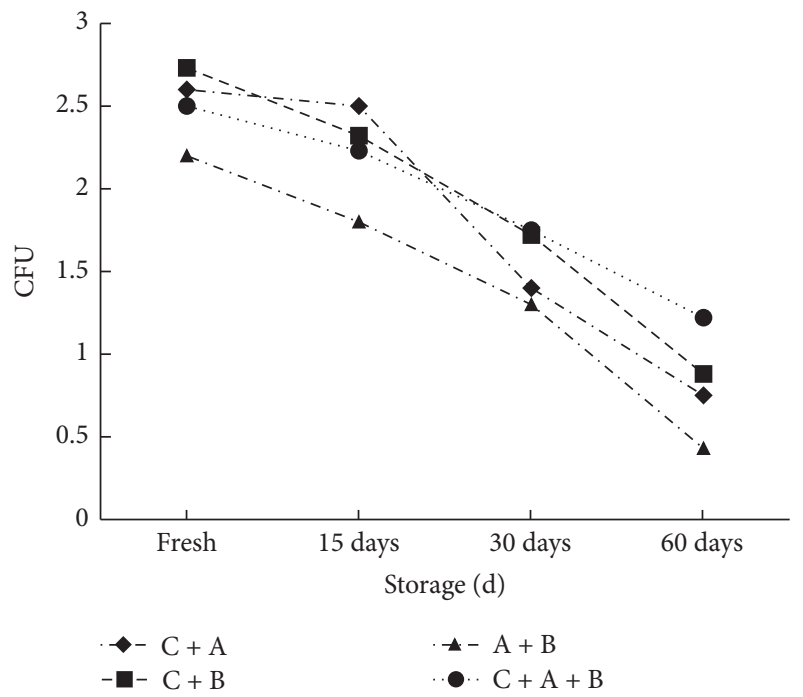

FIgURE 7: Changes in viability of Bifidobacterium spp. in frozen yogurt.

to $0.88 \times 10^{8} \mathrm{CFU}$ with decrease percent $76.80 \%$ from fresh to 60 days of storage. Moreover, frozen yogurt made with Bifidobacterium spp. culture of B. adolescentis $+B$. infantis (A + B) had count of $2.2 \times 10^{8}$ to $0.43 \times 10^{8} \mathrm{CFU}$ with decrease percent $80.50 \%$ from fresh to 60 days of storage period. Finally, the count of Bifidobacterium spp. for frozen yogurt mix made with yogurt culture $+B$. adolescentis $+B$. infantis 


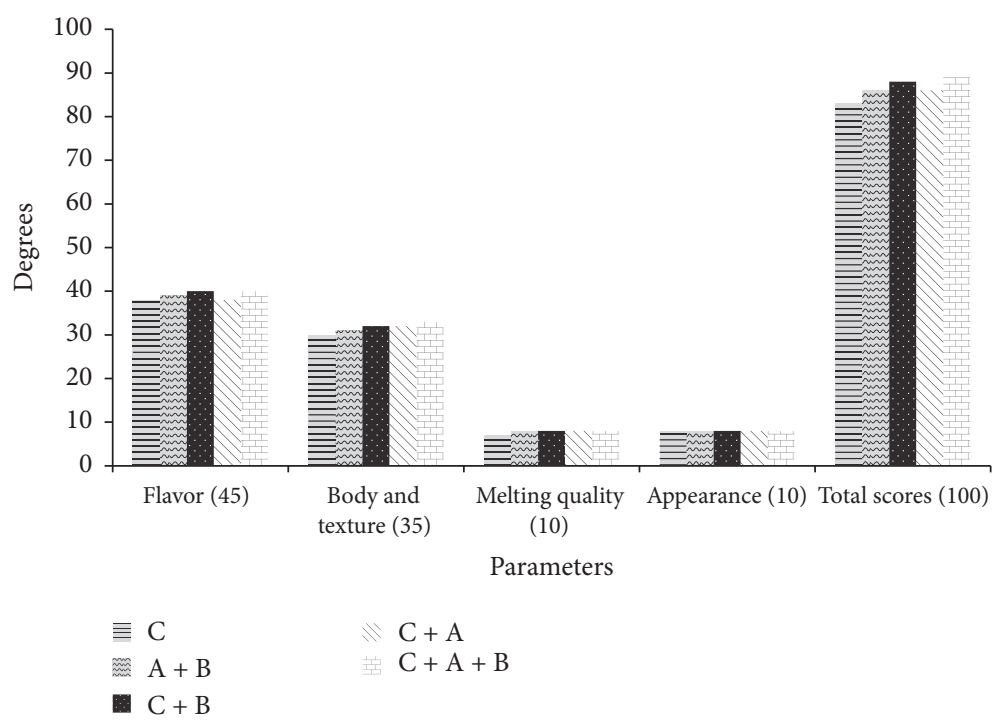

FIGURE 8: Sensory evaluation of frozen yogurt.

$(\mathrm{C}+\mathrm{A}+\mathrm{B})$ was from $2.5 \times 10^{8}$ to $1.22 \times 10^{8} \mathrm{CFU}$ with decrease percent $51.20 \%$ from fresh to 60 days of storage period. These data were in close agreement with data obtained by $[30,33]$ who reported that no significant $(p>0.05)$ difference was observed in the count of yogurt bacteria as well as B. bifidum count between different frozen yogurt mixes.

4.7. Sensory Evaluation of Frozen Yogurt after 60 Days of Storage at $-18^{\circ} \mathrm{C}$. Results in Figure 8 have shown the evaluation scores of frozen yogurt made with yogurt culture and Bifidobacterium spp. after 60 days of storage at $-18^{\circ} \mathrm{C}$. It indicated that there were no high differences between samples in sensory evaluation. It appeared that frozen yogurt made with yogurt culture + Bifidobacterium adolescentis + Bifidobacterium infantis $(\mathrm{C}+\mathrm{A}+\mathrm{B})$ gained a high score of 89 . In addition, samples made with yogurt culture + Bifidobacterium infantis $(\mathrm{C}+\mathrm{B})$ gained score of 88 in total as well.

There are at least two important aspects that should be highlighted while analyzing frozen yogurt. First, consumers are used to the flavor of dairy products produced with traditional yogurt bacteria, which would lead to lower sensory scores to products that do not fit into this category. Secondly, Bifidobacterium spp. are heterofermentative organisms, which are able to produce several types of organic acids (lactic, acetic, and formic acid) and ethanol [34] which can induce important flavor modifications. Considering the potential benefits provided by the probiotic microorganisms, process adjustments could be implemented in order to overcome any possible flavor or aroma issues. In spite of the slightly acidic flavor of their samples, unfamiliar to our consumers, all these samples were acceptable. The results in Figure 9 closely agreed with results obtained by [35], who found that the overall acceptance of probiotic ice cream depends on the preferred and accepted $\mathrm{pH}$.

Results of the PCA were used to analyze physicochemical characteristics, some rheological properties, and sensory

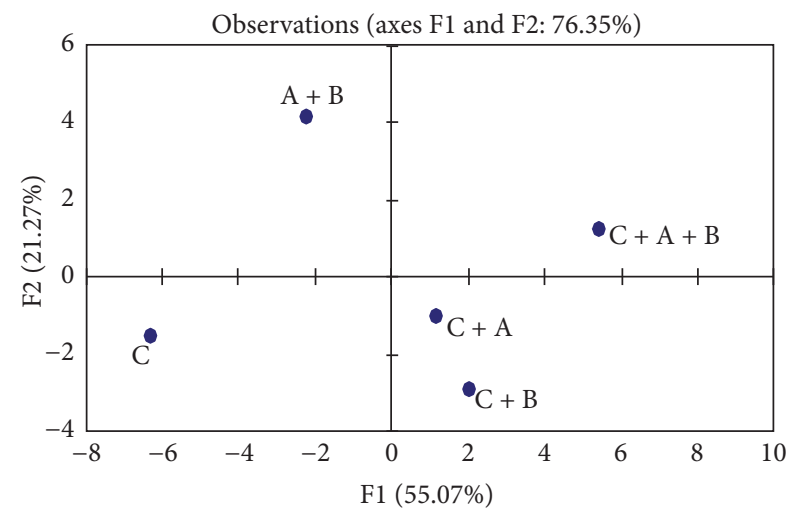

FIGURE 9: Plots of the $x$-loadings.

evaluation of frozen yogurts. Figure 9 presented the correlation loadings. The scores plot of PCA illustrated the large variability of five mixes of frozen yogurt based on different species of Bifidobacterium spp. during 60 days of storage at $-18^{\circ} \mathrm{C}$. Loadings were the coefficients of the original variables of each principal component. Inertia percentage and correlated variables for axes 1 and 2 were displayed in Table 3. Axis 1 explained $55.08 \%$ of the total inertia. Axis 2 explained $21.27 \%$ of the inertia. With respect to the explanatory variables, Figure 9 shows four clusters of mixes. The first cluster included the $\mathrm{C}+\mathrm{A}$ and $\mathrm{C}+\mathrm{B}$, whereas the second, third, and fourth clusters were $\mathrm{C}, \mathrm{A}+\mathrm{B}$, and $\mathrm{C}+\mathrm{A}+$ $\mathrm{B}$, respectively, individualized.

\section{Conclusion}

Bifidobacterium spp. can grow well and have ability to withstand different conditions of acidity and bile. Moreover, frozen yogurt can serve as an excellent vehicle for dietary incorporation of probiotic bacteria. On the contrary, frozen 
TABLE 3: Discriminate variable factors of principal components analyses of analyzed physicochemical characteristics, some rheological properties, and sensory evaluation.

\begin{tabular}{lcc}
\hline & F1 & F2 \\
\hline Proper value & 15.97 & 6.17 \\
Variability (\%) & 55.08 & 21.27 \\
Cumulative (\%) & 55.08 & 76.35 \\
\hline
\end{tabular}

storage of the products has little effects on the survival of Bifidobacterium spp., which are sufficient to offer the suggested therapeutic effects. Supplementation with Bifidobacterium spp. has been found to exert a little effect on flavor or compositional characteristics of frozen yogurt. Our previous study indicated that there were no significant difference changes $(p>0.05)$ during adding different Bifidobacterium spp. in the physiochemistry or sensory evaluation of frozen yogurt.

\section{Conflicts of Interest}

The authors declare that there are no conflicts of interest regarding the publication of this study.

\section{References}

[1] C. Soukoulis, I. D. Fisk, and T. Bohn, "Ice cream as a vehicle for incorporating health-promoting ingredients: conceptualization and overview of quality and storage stability," Comprehensive Reviews in Food Science and Food Safety, vol. 13, no. 4, pp. 627655, 2014.

[2] M. K. Tripathi and S. K. Giri, "Probiotic functional foods: survival of probiotics during processing and storage," Journal of Functional Foods, vol. 9, no. 1, pp. 225-241, 2014.

[3] N. Saad, C. Delattre, M. Urdaci, J. M. Schmitter, and P. Bressollier, "An overview of the last advances in probiotic and prebiotic field," LWT - Food Science and Technology, vol. 50, no. 1, pp. 1-16, 2013.

[4] M. E. Sanders, F. Guarner, R. Guerrant et al., "An update on the use and investigation of probiotics in health and disease," Gut, vol. 62, no. 5, pp. 787-796, 2013.

[5] V. Grimm, C. Westermann, and C. U. Riedel, "Bifidobacteriahost interactions-an update on colonisation factors," BioMed Research International, vol. 2014, Article ID 960826, 10 pages, 2014.

[6] R. Tojo, A. Suarez, and M. G. Clemente, "Intestinal microbiota in health and disease: role of bifidobacteria in gut homeostasis," World Journal of Gastroenterology, vol. 20, no. 41, pp. 1516315176, 2014

[7] P. H. P. Prasanna, A. S. Grandison, and D. Charalampopoulos, "Screening human intestinal Bifidobacterium strains for growth, acidification, EPS production and viscosity potential in low-fat milk," International Dairy Journal, vol. 23, no. 1, pp. 3644, 2012.

[8] P. H. P. Prasanna, A. S. Grandison, and D. Charalampopoulos, "Effect of dairy-based protein sources and temperature on growth, acidification and exopolysaccharide production of Bifidobacterium strains in skim milk," Food Research International, vol. 47, no. 1, pp. 6-12, 2012.
[9] T. R. Pugazhenthi, A. Elango, and D. Vijaya, "Dietetic frozen probiotic yogurt - preparation and its evaluation," in Proceedings of 6 th international conference on emerging technologies in food and nutrition for health management, vol. 4, 2, pp. 2320-7876, 2015, http://www.ijfans.com/.

[10] M. C. Silva, V. B. D. Souza, M. Thomazini et al., "Use of the jabuticaba (Myrciaria cauliflora) depulping residue toproduce a natural pigment powder with functional properties," $L W T$ Food Science and Technology, vol. 55, no. 1, pp. 203-209, 2014.

[11] A. Medeiros, M. Thomazini, A. Urbano, R. Correia, and C. Favaro-Trindade, "Structural characterization and cell viability of a spray dried probiotic yogurt produced with goats' milk and Bifidobacterium animalis subsp. lactis BI-07," International Dairy Journal, vol. 39, pp. 71-77, 2014.

[12] J. L. Ferraz, A. G. Cruz, R. S. Cadena et al., "Sensory acceptance and survival of probiotic bacteria in ice cream produced with different overrun levels," Journal of Food Science, vol. 77, no. 1, pp. 24-28, January 2012.

[13] A. A. Al-Saleh, A. S. Zahran, and H. M. Abu-Tarboush, "Growth of bifidobacteria: environmental conditions and adherence to epithelial cells," Milchwissenschaft, vol. 53, no. 4, 1998.

[14] R. Khalil, H. Mahrous, K. El-Halafawy, K. Kamaly, J. Frank, and M. El Soda, "Evaluation of the probiotic potential of lactic acid bacteria isolated from faeces of breast-fed infants in Egypt," African Journal of Biotechnology, vol. 6, no. 7, pp. 939-949, 2007.

[15] D. K. Walker and S. E. Gilliland, "Relationship among bile tolerance, bile salt deconjugation, and assimilation of cholesterol by Lactobacillus acidophilus," Journal of Dairy Science, vol. 76, no. 4, pp. 956-961, 1993.

[16] R. L. Bradely and M. Hekmati, "Preparation of frozen yogurt," United Pataent, vol. 4, pp. 293-573, 1981.

[17] M. B. Akin, M. S. Akin, and Z. Kirmaci, "Effects of inulin and sugar levels on the viability of yogurt and probiotic bacteria and the physical and sensory characteristics in probiotic ice-cream," Food Chemistry, vol. 104, no. 1, pp. 93-99, 2007.

[18] M. R. Muse and R. W. Hartel, "Ice cream structural elements that affect melting rate and hardness," Journal of Dairy Science, vol. 87, no. 1, pp. 1-10, 2004.

[19] P. N. Rossa, V. M. Burin, and M. T. Bordignon-Luiz, "Effect of microbial transglutaminase on functional and rheological properties of ice cream with different fat contents," LWT - Food Science and Technology, vol. 48, no. 2, pp. 224-230, 2012.

[20] H. Magariños, S. Selaive, M. Costa, M. Flores, and O. Pizarro, "Viability of probiotic micro-organisms (Lactobacillus acidophilus La-5 and Bifidobacterium animalis subsp. lactis Bb-12) in ice cream," International Journal of Dairy Technology, vol. 60, no. 2, pp. 128-134, 2007.

[21] S. I. Farag, A. E. Khader, A. M. Moussa, and A. M. El-Batawy, "A study on ice cream. I.On the use of high fructose syrup as a sweetener," Egyptian Journal of Dairy Science, vol. 21, no. 1, pp. 97-107, 1993.

[22] R. P. K. Sahadeva, S. F. Leong, K. H. Chua et al., "Survival of commercial probiotic strains to $\mathrm{pH}$ and bile," International Food Research Journal, vol. 18, no. 4, pp. 1515-1522, 2011.

[23] H. S. Chung, Y. B. Kim, S. L. Chun, and G. E. Ji, "Screening and selection of acid and bile resistant bifidobacteria," International Journal of Food Microbiology, vol. 47, no. 1-2, pp. 25-32, 1999.

[24] S. Kaewnopparat, N. Dangmanee, N. Kaewnopparat, T. Srichana, M. Chulasiri, and S. Settharaksa, "Invitro probiotic properties of Lactobacillus fermentum SK5 isolated from vagina ofa healthy woman," Anaerobe, vol. 22, pp. 6-13, 2013. 
[25] A. EL-Shazly, M. A. EL-Tahra, and M. M. Abo-Sera, "Effect of different methods for the manufacture of frozen yogurt on its properties," in Proceedings of the 9th Egyptian Inter. Conference of Dairy Science \& Technology, Milk and product for a healthy future, 2004.

[26] A. Abghari, M. Sheikh-Zeinoddin, and S. Soleimanian-Zad, "Nonfermented ice cream as a carrier for Lactobacillus acidophilus and Lactobacillus rhamnosus," International Journal of Food Science and Technology, vol. 46, no. 1, pp. 84-92, 2011.

[27] K. M. K. Kebary, "Viability of Bifidobacterium bifidum and its effect on quality of frozen Zabady," Food Research International, vol. 29, no. 5-6, pp. 431-437, 1996.

[28] C. Senaka Ranadheera, C. A. Evans, M. C. Adams, and S. K. Baines, "Production of probiotic ice cream from goat's milk and effect of packaging materials on product quality," Small Ruminant Research, vol. 112, no. 1-3, pp. 174-180, 2013.

[29] E. Mahdian and R. Karazhian, "Effects of fat replacers and stabilizers on rheological, physicochemical and sensory properties of reduced-fat ice cream," Journal of Agricultural Science and Technology, vol. 15, no. 6, pp. 1163-1174, 2013.

[30] P. D. L. D. Silva, M. D. F. Bezerra, K. D. Santos, and R. Correia, "Potentially probiotic ice cream from goat's milk: characterization and cell viability during processing, storage and simulated gastrointestinal conditions," LWT-Food Science and Technology, vol. 62, no. 1, pp. 452-457, 2015.

[31] T. Erkaya, E. Dagdemir, and M. Şengül, "Influence of Cape gooseberry (Physalis peruviana L.) addition on the chemical and sensory characteristics and mineral concentrations of ice cream," Food Research International, vol. 45, no. 1, pp. 331-335, 2012.

[32] S. Pinto, C. Fritzen-Freire, I. MuuNoz, P. Barreto, E. Prudêncio, and R. Amboni, "Effects of the addition of microencapsulated Bifidobacterium BB-12 on the properties of frozen yogurt," Journal of Food Engineering, vol. 11, pp. 563-569, 2012.

[33] T. R. Pugazhenthi, A. Elango, D. Vijaya, and V. Jayalalitha, Preparation and Evaluation of Dietetic Frozen Probiotic Yogurt, Probiotics in Sustainable Food Production: Current Status and Future Prospects-Probiotic Foods, ISBN 978-93-82338-47-5, 2013.

[34] H. Jalili, S. Razavi, M. Safari, and F. Malcata, "Enhancement of growth rate and $\beta$-galactosidase activity, and variation in organic acid profile of Bifidobacterium animalis subsp. lactis Bb 12, Enzyme and Microbial Technology, vol. 45, no. 6-7, pp. 469476, 2009.

[35] S. Hekmat and D. J. McMahon, "Survival of Lactobacillus acidophilus and Bifidobacterium bifidum in ice cream for use as a probiotic food." Journal of dairy science, vol. 75, no. 6, pp. 14151422, 1992. 

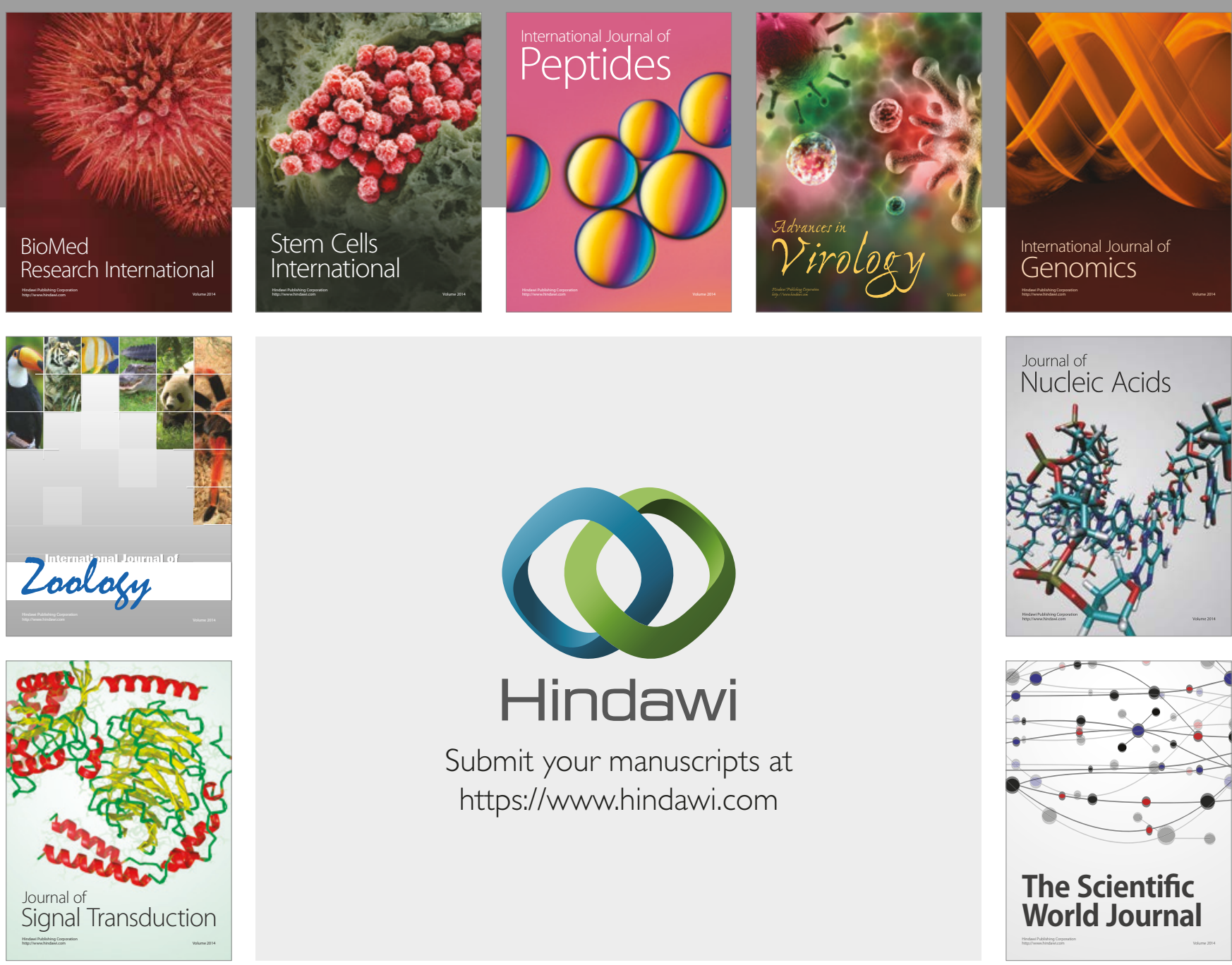

Submit your manuscripts at

https://www.hindawi.com
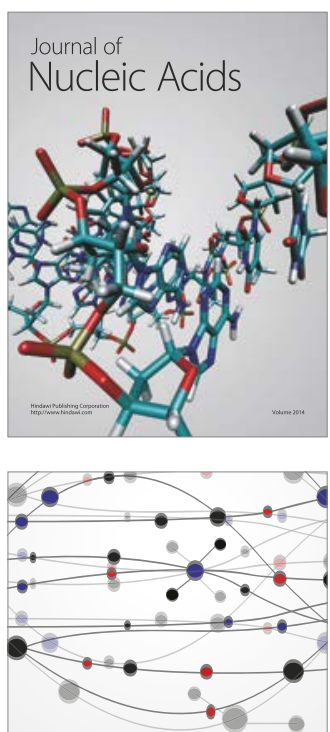

The Scientific World Journal

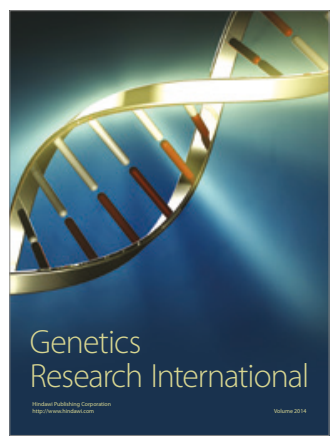

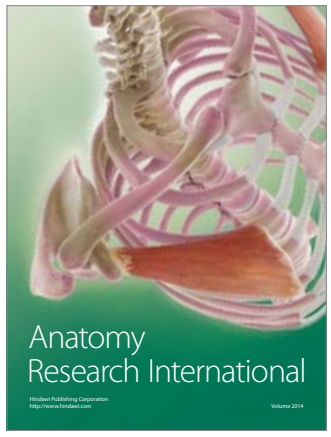

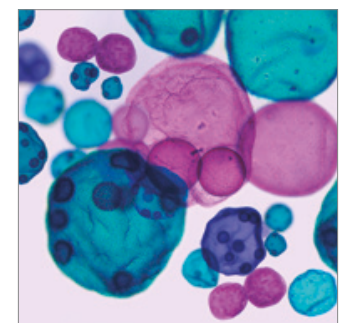

International Journal of Microbiology
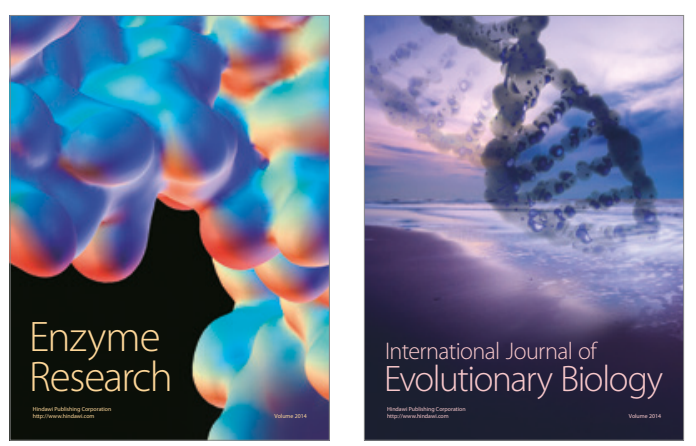
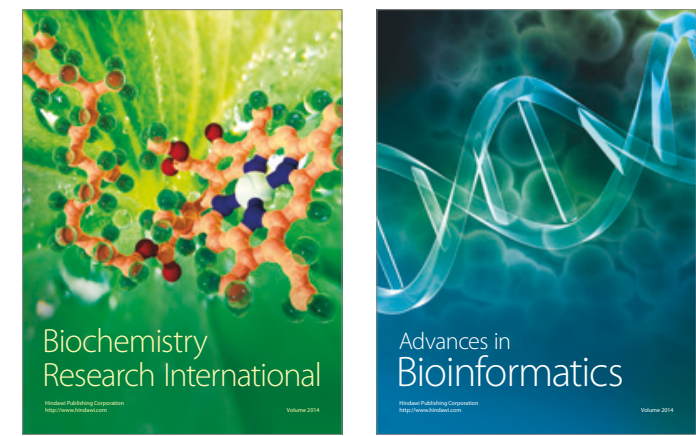

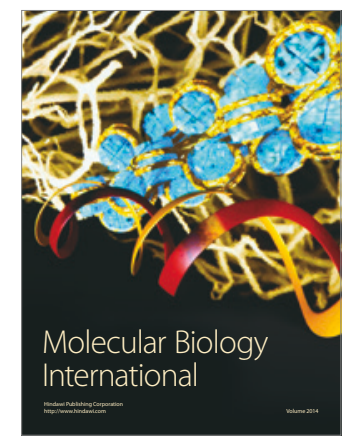

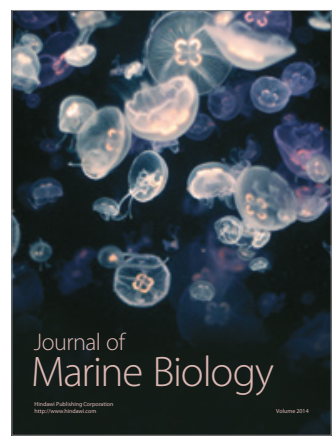

